\title{
Cooperative Jamming via Spectrum Leasing
}

\author{
Igor Stanojev and Aylin Yener \\ The Pennsylvania State University, University Park, PA 16802 \\ ixs11@psu.edu, yener@ee.psu.edu
}

\begin{abstract}
Secure communication rates can be facilitated or enhanced via deployment of cooperative jammers in a multiterminal environment. Such an approach typically assumes dedicated and/or altruistic jamming nodes, investing their resources for the good of the whole system. In this paper, we demonstrate that jammers can be recruited to provide significant improvements of secrecy rates even when this assumption is alleviated. A game-theoretic framework is proposed where a source node, towards the maximization of its secrecy rate, utilizes the jamming services from a set of non-altruistic nodes, compensating them with a fraction of its bandwidth for transmission of their user data. With the goal of maximizing their user-data transmission rate priced by the invested power, potential cooperative jammers will provide the jamming/transmitting power that is generally proportional to the amount of leased bandwidth. Elaborating initially on a single-jammer scenario, interaction between the source and a cooperative jammer is modeled as the Stackelberg leader-follower game. The scheme is further extended to involve multiple potential jammers, applying competition mechanisms such as the auctioning and power control game, while maintaining the Stackelberg framework.
\end{abstract}

\section{INTRODUCTION}

Among the most concerning implications of the broadcast nature of a wireless medium is its vulnerability to eavesdropping. Recent efforts have, nevertheless, shown that the broadcast nature can also be exploited to counter eavesdropping and improve the rate at which secret communication can take place. In particular, deploying cooperative jammers that transmit Gaussian noise [5] [6] or jamming codewords [7][9] can help in impairing the reception of the eavesdropper(s), thus enhancing secure communication rates between legitimate parties.

Possible secrecy gains acquired via the cooperative jamming paradigm, as was first proposed in [5], generally come with the assumption of altruistic and/or dedicated jamming nodes [5]-[12], willing to unconditionally utilize their resources for the benefit of the system performance. The validity of this assumption can be questioned, especially for mobile devices with limited batteries. To address this issue, we propose a game-theoretic framework wherein the non-altruistic nodes, i.e., nodes with their own data, can facilitate secure sourcedestination communication by taking the role of cooperative jammers, if appropriately compensated through a spectrum lease that would enable them to transmit their own data. The scheme is motivated by the recently proposed spectrum leasing for cooperation paradigm [13] [14], wherein a similar setting is applied, with non-altruistic nodes performing the role of a relay instead of a cooperative jammer.
Focusing initially on the reference case with a single potential jammer, the proposed scheme is outlined as follows. A source communicates with its destination in the presence of an eavesdropper and a separate non-altruistic node. The source is willing to compensate the non-altruistic node for its cooperative jamming services with an access to a fraction of its transmission interval/bandwidth. In doing so, the source aims to maximize its secrecy rate, i.e., the rate at which no information is revealed to the eavesdropper. On the other hand, the potential jammer optimizes its power with the goal of maximizing its data transmission rate during the leased interval, discounted by the cost of the overall transmitted power. The jammer is constrained by the amount of leased resource and a requirement to use the same power during both transmitting and jamming phases. This interaction between the source and a potential non-altruistic jammer is conveniently cast in the framework of Stackelberg game [15], with source as the spectrum owner in the role of the game leader and jammer as the follower, and its outcome is the Stackelberg equilibrium, as elaborated in Section III.

The scheme is further extended to involve multiple potential jammers, modeling their competition for bandwidth access via distributed resource allocation mechanisms such as auctioning [16] [14] in Section IV, and power control game [18] [13] in Section V. Interaction between the source and the set of potential cooperative jammers thus becomes a two-layer game, with a Stackelberg game described above as the 'outer' framework, while a set of jammers constitutes the follower entity whose power response is the outcome of the auction or power control game played between jammers, with the maximization goals for both mechanisms as described above.

It is noted that, in addition to facilitating an interesting framework for the deployment of cooperative jamming paradigm via spectrum leasing, the proposed solution can be alternatively applied as a practical solution for cognitive radio networks operating according to property-rights model [21]. In such networks, primary, i.e., licensed users may lease portions of the licensed spectrum to secondary, i.e., unlicensed users in exchange for some form of compensation. Here, the role of the primary node is played by the source transmitting a confidential message and that of the secondary by the jamming nodes. Moreover, retribution from secondary to primary nodes is in the form of cooperative jamming to the primary secret transmission. This enables on-the-air decisions and avoids the regulatory issues or money transactions that commonly hinder the implementation of the property-rights spectrum leasing concept. 


\section{System PARAMETERS AND NOTATION}

We consider a scenario where source $\mathrm{S}$ communicates with destination $\mathrm{D}$ using bandwidth/time-slot normalized to unity, in the presence of an eavesdropper $\mathrm{E}$ from whom the communication must be kept secret. There are $N$ additional nodes $\mathrm{J}_{i}, i=1, \ldots, N$ present, each of which has data to transmit towards its intended receiver $\mathrm{D}_{\mathrm{J}, i}$ and can act as potential cooperative jammer for the S-D secure communication, as illustrated in Figure 1 for $N=1$. The channel gains between nodes are modeled as independent complex Gaussian random variables. Instantaneous power channel gains between node $\mathrm{S}$ and nodes $\mathrm{D}$ and $\mathrm{E}$ are denoted as $h_{\mathrm{SD}}$ and $h_{\mathrm{SE}}$, respectively, between node $\mathrm{J}_{i}$ and nodes $\mathrm{D}$ and $\mathrm{E}$ as $h_{\mathrm{J}_{i} \mathrm{D}}$ and $h_{\mathrm{J}_{i} \mathrm{E}}$, respectively, and between $\mathrm{J}_{i}$ and $\mathrm{D}_{\mathrm{J}, l}$ as $h_{\mathrm{J}_{i l}}, i, l=1, . ., N$. Average transmission power for the source $\mathrm{S}$ is $P_{\mathrm{S}}$, while the average transmission/jamming power of $\mathrm{J}_{i}$ is $P_{\mathrm{J}_{i}}$, limited by the power budget $P_{\mathrm{J}_{i}} \leq \bar{P}_{\mathrm{J}_{i}}$. The independent additive white Gaussian noise variance for each link is $\sigma^{2}$. For the case with $N=1$ potential jammer, index is removed from notation for clarity. Throughout the paper, we assume signaling using Gaussian codebooks and the cooperative jamming in the form of Gaussian noise.

\section{REFERENCE STACKELBERG MOdEL}

In this section, a reference scheme with $N=1$ potential jammers is presented. The communication model is introduced in Section III-A and then presented in the Stackelberg framework in Section III-B. A straightforward extension of the reference Stackelberg scheme to a multi-jammer scenario is given in Section III-C.

\section{A. Reference Communications Model}

The scenario of interest is illustrated in Figure 1 and involves the source $\mathrm{S}$ communicating secretly with the destination $\mathrm{D}$ in the presence of eavesdropper $\mathrm{E}$, looking to recruit node $\mathrm{J}$ as a cooperative jammer, if it is potentially helpful to increase its secrecy rate. In particular, the source is willing to compensate the potential jammer with a bandwidth fraction $1-\alpha$ for jammer's own data transmission, as in Figure 1-(a), while preserving the remaining bandwidth $\alpha$ for its secret communication aided by cooperative jamming, as in Figure 1-(b). During the cooperative jamming phase, node J is required to transmit Gaussian noise with the same power $P_{\mathrm{J}}$ used during its data transmission. This rule is enforced in order to ensure that the jammer spends for cooperation jamming the same power that they are willing to spend for their own transmission. The achievable S-D secrecy rate, that is the communication rate at which no information is revealed to the eavesdropper, assisted by cooperative jamming is given by [5] [6]:

$$
\begin{aligned}
R_{\mathrm{S}}\left(\alpha, P_{\mathrm{J}}\right)= & \alpha\left[\log _{2}\left(1+\frac{h_{\mathrm{SD}} P_{\mathrm{S}} / \alpha}{\sigma^{2}+h_{\mathrm{JD}} P_{\mathrm{J}}}\right)-\right. \\
& \left.\log _{2}\left(1+\frac{h_{\mathrm{SE}} P_{\mathrm{S}} / \alpha}{\sigma^{2}+h_{\mathrm{JE}} P_{\mathrm{J}}}\right)\right]^{+},
\end{aligned}
$$

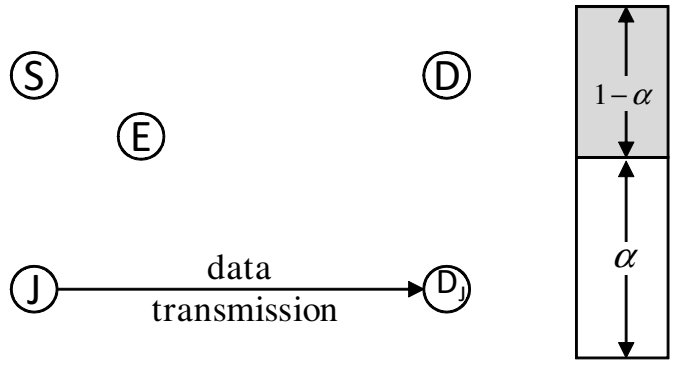

(a)

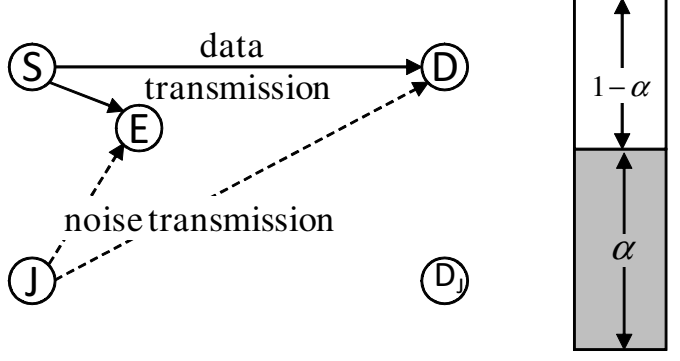

(b)

Figure 1. Example of spectrum leasing for cooperative jamming with $N=1$ cooperative jammers.

where $[x]^{+}=\max (0, x)$, while the source transmission power $P_{\mathrm{S}}$ is normalized with parameter $\alpha$ to satisfy the average transmission power constraint. The utility of the node acting as cooperative jammer is defined as its rate during the fraction $1-\alpha$ towards its destination $\mathrm{D}_{\mathrm{J}}$, priced by the cost of the transmission power [13]:

$$
U_{\mathrm{J}}\left(\alpha ; P_{\mathrm{J}}\right)=(1-\alpha) \log \left(1+\frac{h_{\mathrm{J}} P_{\mathrm{J}}}{\sigma^{2}}\right)-c P_{\mathrm{J}},
$$

where $c$ is the cost per unit transmission power and $P_{\mathrm{J}} \leq$ $\bar{P}_{\mathrm{J}}$. Notice that the jammer transmission power $P_{\mathrm{J}}$ requires no normalization, as the jammer transmits during the entire transmission interval, as illustrated in Figure 1.

It was determined in [6] that the following conditions are required for the cooperative jamming to yield improvement on the secrecy rate:

$$
\begin{aligned}
\frac{h_{\mathrm{SE}} h_{\mathrm{JD}}}{h_{\mathrm{SD}} h_{\mathrm{JE}}} & <1 \\
\frac{h_{\mathrm{SD}} h_{\mathrm{JD}}\left(\sigma^{2}+h_{\mathrm{SE}} P_{\mathrm{S}} / \alpha\right)}{h_{\mathrm{SE}} h_{\mathrm{JE}}\left(\sigma^{2}+h_{\mathrm{SD}} P_{\mathrm{S}} / \alpha\right)} & <1 .
\end{aligned}
$$

Moreover, the jammer utility (2) is clearly concave and negative when $P_{\mathrm{J}} \rightarrow \infty$. For such a function to have a positive value over some range of $P_{\mathrm{J}}$, the condition $\partial U_{\mathrm{J}} / \partial P_{\mathrm{J}}>0$ must hold:

$$
h_{\mathrm{J}}>N_{0} /(1-\alpha) c \ln 2 .
$$

With this condition, utility $U_{\mathrm{J}}$ is positive for $P_{\mathrm{J}} \in$ $\left(0, P_{\mathrm{J}}^{\lim }(\alpha)\right)$, where $P_{\mathrm{J}}^{\lim }(\alpha)$ is the positive solution of 
$U_{\mathrm{J}}\left(\alpha, P_{\mathrm{J}}(\alpha)\right)=0$ and reads:

$$
P_{\mathrm{J}}^{\lim }(\alpha)=\frac{N_{0}}{h_{\mathrm{J}}}\left(-\frac{1}{k(\alpha)} \mathcal{W}_{-1}\left(-k(\alpha) e^{k(\alpha)}\right)-1\right),
$$

where $k(\alpha)=c \ln 2 \cdot \sigma^{2} / h_{\mathrm{J}} /(1-\alpha)$ and $\mathcal{W}_{l}(x)$ is is the $l$ th branch of the multi-valued Lambert $\mathrm{W}$ function [22].

\section{B. Game-Theoretic (Stackelberg) Model}

Throughout this work, the nodes are defined as selfish and rational [15] to mimic a non-altruistic behavior. An appropriate framework for analyzing the interaction between such nodes is game theory [15]. In particular, a convenient setting here is that of the Stackelberg game [15], wherein one agent, termed follower, acts subject to the strategy chosen by the other agent, leader, which in turns seeks maximization of its own utility. Here, the game leader and the follower are the source and the cooperative jammer, respectively. The source's optimal strategy $\alpha^{*}$ and the corresponding power choice of the jammer $P_{\mathrm{J}}^{*}\left(\alpha^{*}\right)$ are jointly referred to as the Stackelberg equilibrium.

Interaction between the source and the potential nonaltruistic jammer is shown in Figure 2. The jammer is aware of parameter $\alpha$ and optimizes its power towards the goal of maximizing its utility, given by (2). The solution of jammer's problem

$$
\begin{gathered}
P_{\mathrm{J}}^{*}(\alpha)=\arg \max _{P_{\mathrm{J}}(\alpha)} U_{\mathrm{J}}\left(\alpha ; P_{\mathrm{J}}(\alpha)\right) \\
\text { s.t. } 0 \leq P_{\mathrm{J}} \leq \bar{P}_{\mathrm{J}},
\end{gathered}
$$

is given as

$$
P_{\mathrm{J}}^{*}(\alpha)=\left[\frac{1-\alpha}{c \ln 2}-\frac{\sigma^{2}}{h_{\mathrm{J}}}\right]_{0}^{\bar{P}_{\mathrm{J}}}
$$

where $[x]_{x_{\min }}^{x_{\max }}=\min \left(\max \left(x_{\min }, x\right), x_{\max }\right)$. Notice from (8) that the power pricing mechanism prevents the source from preserving an unfairly large amount of bandwidth $\alpha$, as this would in turn typically implicate a small cooperative jamming power $P_{\mathrm{J}}^{*}(\alpha)$ or even lead to a denial of jamming participation $\left(P_{\mathrm{J}}^{*}(\alpha)=0\right)$.

On the other hand, the source, acting as the game leader, determines the fraction $\alpha$ towards the goal of maximizing its secrecy rate (1), knowing that its decision will affect the strategy selected by the jammer:

$$
\begin{gathered}
\alpha^{*}=\arg \max _{\alpha} R_{\mathrm{S}}\left(\alpha, P_{\mathrm{J}}^{*}(\alpha)\right) \\
\text { s.t. } 0<\alpha \leq 1,
\end{gathered}
$$

where $P_{\mathrm{J}}^{*}(\alpha)$ is given by (8). Equations (8) and (9) constitute the Stackelberg equilibrium for the described model. Furthermore, (9) includes the possibility of refusing the jammer's cooperation, $\alpha=1$, if the latter is not contributing, in which case the jammer's utility is zero. The source is assumed to have the complete knowledge of all the channel gains in the system, while the knowledge of $h_{\mathrm{J}}$ is required at the jammer. Albeit ideal, the assumption of instantaneous Channel State Information (CSI) at transmitters is common in the literature on game-theoretic applications to wireless networks [13] [18]

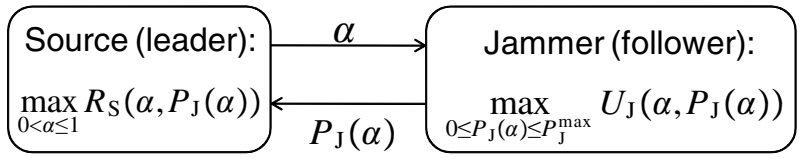

Figure 2. Stackelberg interaction between the source and the potential cooperative jamming node.

and provides a benchmark for analysis. As a final remark, we consider the scenario where nodes who agree on being cooperative jammers are honest and trusted, i.e., they do not deviate from the expected behavior of jamming with the same power, nor turn malicious.

\section{Application to Multi-Jammer Scenario}

The framework described above can also be used for the environment involving multiple potential cooperative jammers. In addition to the parameter $\alpha$, the source would also have to indicate the index of the 'winning' jammer, i.e., the one that would mostly contribute to the source's secrecy rate. Although legitimate, this approach fails to fully exploit the selfish and rational features of potential jammers, which are likely to lead to contention for access to the bandwidth and consequently further improvement for the source [15]. In the following two sections, we elaborate on two mechanisms that incorporate the competitive jammers' behavior.

\section{Auction Scheme}

This section introduces the scheme involving multiple potential cooperative jammers $\mathrm{J}_{i}, i=1, . ., N$, competing to gain the spectrum access for transmission of their user data towards their single intended receiver $\mathrm{D}_{\mathrm{J}, i}$, by offering cooperative jamming services to the source. The communication model and the interaction between the source and the set of potential jammers follow the lines of Section III, with the difference that the follower entity is now a set of $N$ jammers and the follower's response $P_{\mathrm{J}_{w(\alpha)}}^{*}(\alpha)$ is the power outcome of the auction game played among jammers, with $w(\alpha)=1, . ., N$ indicating the winning cooperative jammer, if any, for a source's strategy $\alpha$, as detailed in the following. The competition model is built upon the rules of Vickrey auction, i.e., the sealed-bid second-price auction [23] [16]. It is noted that the considered framework is, however, not limited to Vickrey auctions and can be implemented via other auction types.

\section{A. Vickrey Auction}

Auctions are a widely accepted mechanism for distribution of limited amount of resources among competing users [16]. Among various auction types, the sealed bid secondprice Vickrey auction, prescribing that the winning bidder is awarded with the bidding item at the price of the second largest bid, is of particular interest, due to its 'truthful bidding' property [23] [16]. Namely, bidders are motivated to bid with the maximum amount they would be willing to pay for the object. Importantly, such a strategic choice corresponds to the game-theoretic concept of dominant strategy equilibrium 
$(D S E)$, defined as the state wherein the strategies are required to remain preferable to every player irrespective of the amount of information available on the other players [15]. This property implies that the bidders require no information on other bidders' strategies or their evaluations of the bidding item. The desirable properties of Vickrey auction have already proved useful in wireless communication problems, see for example [14].

\section{B. Modified Vickrey Auction for Communication Model}

Here, the auctioneer's utility is defined as the secrecy rate $R_{\mathrm{S}}$ as in (1), a bidder $\mathrm{J}_{i}$ 's strategy is its power $P_{\mathrm{J}_{i}}$ and a bidder's utility $U_{\mathrm{J}_{i}}$ is defined in (2), given that it is granted the spectrum access, i.e., that it won the auction, and zero otherwise. For a given $\alpha$, the bids are in the form of the secrecy rate $R_{\mathrm{S}}\left(\alpha, P_{\mathrm{J}_{i}}\right)$, implying that a bidder $\mathrm{J}_{i}$ must be aware of the source's parameters $h_{\mathrm{SD}}$ and $h_{\mathrm{SE}}$. The source sets the lowest acceptable rate, i.e., the reserve price, as $R_{S}\left(\alpha, P_{\mathrm{J}}=0\right)$, accepting only larger bids. Denoting the bidding strategies in the DSE equilibrium as $P_{\mathrm{J}_{i}}^{\text {bid }}$, the index of a winning cooperative jammer for a given $\alpha$ is $w(\alpha)=\arg \max _{i=1, \ldots, N} R_{\mathrm{S}}\left(\alpha, P_{\mathrm{J}_{i}}^{\mathrm{bid}}(\alpha)\right)$, if $R_{\mathrm{S}}\left(\alpha, P_{\mathrm{J}_{w(\alpha)}}^{\text {bid }}\right)>R_{S}(\alpha, 0)$, otherwise no jammer is chosen. The standard assumption, also adopted here, is that, in the case of multiple equal highest offers, the situation is resolved by random allotment to one of them. Furthermore, the secondbest rate $R_{\mathrm{S}, 2}$ reads:

$$
R_{\mathrm{S}, 2}(\alpha)=\max \left(\max _{i \neq w(\alpha)} R_{\mathrm{S}}\left(\alpha, P_{\mathrm{J}_{i}}^{\mathrm{bid}}(\alpha)\right), R_{\mathrm{S}}(\alpha, 0)\right) .
$$

Before proceeding to Section IV-C to determine the DSE and the Stackelberg equilibria, we notice that the auctioning analytical tools are developed for relatively simple linear or monotonic utility functions [16], which might not be the case for the model herein. On this line, we borrow the result from [6] that establishes the impact $P_{\mathrm{J}_{i}}$ on $R_{\mathrm{S}}\left(\alpha, P_{\mathrm{J}_{i}}\right)$ for a given $\alpha$ and formulate it as the following lemma. As discussed below, the lemma indicates that it is possible to improve the DSE equilibrium for the communication model at hand and also provides a guideline to finding the auction bidding equilibrium $P_{\mathrm{J}_{i}}^{\text {bid }}(\alpha), w(\alpha)$ and the auction outcome $P_{\mathrm{J}_{w(\alpha)}^{*}}^{*}(\alpha)$ in Section IV-C.

Lemma 1. [6] For a given $\alpha$, the function $R_{S}\left(\alpha, P_{\mathrm{J}_{i}}\right)$ is quasi-concave in $P_{\mathrm{J}_{i}}$, for $P_{\mathrm{J}_{i}} \geq 0$, with the maximum at $P_{\mathrm{J}_{i}}^{\max }=\arg \max _{P_{\mathrm{J}_{i}}} R_{\mathrm{S}}\left(\alpha, P_{\mathrm{J}_{i}}\right)>0$ given by

$$
\begin{aligned}
P_{\mathrm{J}_{i}}^{\max }(\alpha)= & \frac{\sigma^{2}\left(h_{\mathrm{SE}}-h_{\mathrm{SD}}\right)}{h_{\mathrm{SD}} h_{\mathrm{J}_{i} \mathrm{E}}-h_{\mathrm{SE}} h_{\mathrm{J}_{i} \mathrm{D}}}+ \\
& \sqrt{\frac{\sigma^{2} h_{\mathrm{SE}} h_{\mathrm{SD}}\left(h_{\mathrm{J}_{i} \mathrm{E}}-h_{\mathrm{J}_{i} \mathrm{D}}\right)}{h_{\mathrm{J}_{i} \mathrm{D}} h_{\mathrm{J}_{i} \mathrm{E}}\left(h_{\mathrm{SD}} h_{\mathrm{J}_{i} \mathrm{E}}-h_{\mathrm{SE}} h_{\mathrm{J}_{i} \mathrm{D}}\right)}} . \\
& \sqrt{\frac{\sigma^{2}\left(h_{\mathrm{J}_{i} \mathrm{E}}-h_{\mathrm{J}_{i} \mathrm{D}}\right)}{h_{\mathrm{SD}} h_{\mathrm{J}_{i} \mathrm{E}}-h_{\mathrm{SE}} h_{\mathrm{J}_{i} \mathrm{D}}}+\frac{P_{\mathrm{S}}}{\alpha}} .
\end{aligned}
$$

if the conditions (3)-(4) are met.
Lemma 1 reveals the quasi-concavity of source's utility versus the power $P_{\mathrm{J}_{i}}$ which, together with the fact that a jammer's utility (2) is concave in $P_{\mathrm{J}_{i}}$ (for $P_{\mathrm{J}_{i}} \geq 0$ ) brings us to the following observation. Unlike the setting in a baseline auction model, wherein the auctioneer and a bidder's utility are monotonically increasing and decreasing in price, respectively, i.e., a profit for one is a negative surplus to another [16], here, due to their (quasi-)concavity, it is possible that the players' utilities for a particular $P_{\mathrm{J}_{i}}$ have slopes of an equal signum and, thus, not necessarily incompatible goals. This observation leads us to propose the following modification of the Vickrey auction rule that can lead to the performance improvement for all the involved nodes in the communication model at hand.

Definition 1. In the proposed modification of Vickrey auction, the winning bidder is required to provide the secrecy rate that is at least the second largest bid, i.e., $R_{\mathrm{S}}\left(\alpha, P_{\mathrm{J}_{w(\alpha)}^{*}}^{*}(\alpha)\right) \geq$ $R_{\mathrm{S}, 2}(\alpha)$.

The second largest bid $R_{\mathrm{S}, 2}(\alpha)$ is given in (10). Unlike the original Vickrey principle, wherein the winning bidder provides the auctioneer with exactly the second-best price, this modification enables the winning node to choose a larger value if, as a result, its utility will increase. Notice that neither of the involved nodes, i.e., auctioneer and a bidder, are harmed by this deviation from the Vickrey principles, quite the opposite. The benefits due to this modification will be clearly visible in the following subsection.

\section{Equilibria}

The strategy for a bidder in the DSE is given by the following theorem.

Theorem 1. For a given $\alpha$, the dominant bidding strategy $P_{\mathrm{J}_{i}}^{\text {bid }}(\alpha)$ for a bidder $\mathrm{J}_{i}$ is:

$$
P_{\mathrm{J}_{i}}^{\text {bid }}(\alpha)=\min \left(P_{\mathrm{J}_{i}}^{\max }(\alpha), P_{\mathrm{J}_{i}}^{\lim }(\alpha), \bar{P}_{\mathrm{J}_{i}}\right),
$$

where $P_{\mathrm{J}_{i}}^{\lim }(\alpha)$ and $P_{\mathrm{J}_{i}}^{\max }(\alpha)$ are given by (6) and (11), respectively.

Proof: The proof is illustrated in Figure 3 for a case $P_{\mathrm{J}_{i}}^{\lim }(\alpha)<\bar{P}_{\mathrm{J}_{i}}$ and given as follows. Since in the case of winning the auction, the bidder has no influence on the second-best bid, the dominant strategy is to maximize the chance of winning, i.e., to bid with $P_{\mathrm{J}_{i}}^{\max }(\alpha)$ if $P_{\mathrm{J}_{i}}^{\max }(\alpha) \leq \min \left(P_{\mathrm{J}_{i}}^{\lim }(\alpha), \bar{P}_{\mathrm{J}_{i}}\right)$. However, in case of $P_{\mathrm{J}_{i}}^{\max }(\alpha)>\min \left(P_{\mathrm{J}_{i}}^{\lim }(\alpha), \bar{P}_{\mathrm{J}_{i}}\right)$, i.e., if $P_{\mathrm{J}_{i}}^{\max }(\alpha)$ yields a negative bidder's utility or is out of permissible power range set by $\bar{P}_{\mathrm{J}_{i}}$, the bidder chooses $\min \left(P_{\mathrm{J}_{i}}^{\lim }(\alpha), \bar{P}_{\mathrm{J}_{i}}\right)$ as bidding with larger $P_{\mathrm{J}_{i}}$ would increase the chance of winning only if incurring a negative bidder's utility or a power that is out of permissible range, while bidding with smaller power would only decrease the chance of winning.

The theorem is valid independent of whether Definition 1 is applied or not. Having won the auction, the winning cooperative jammer $\mathrm{J}_{w(\alpha)}$ has to provide the transmitting/jamming 


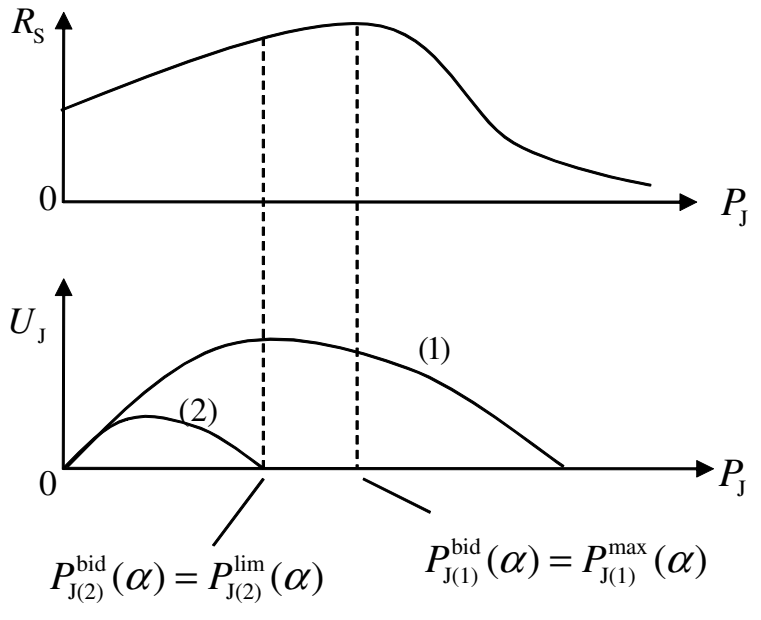

Figure 3. Illustration of Theorem 1.

power that produces at least $R_{\mathrm{S}, 2}(\alpha)$. The following theorem provides this auction outcome.

Theorem 2. Under the rule in Definition 1, the power $P_{J_{w(\alpha)}}^{*}(\alpha)$ chosen by the winning cooperative jammer $\mathrm{J}_{w(\alpha)}$ is given by

$$
P_{\mathrm{J}_{w(\alpha)}}^{*}(\alpha)= \begin{cases}P_{M}, & P_{\mathrm{J}_{w(\alpha)}}^{\mathrm{opt}}(\alpha)>P_{M}, R_{\mathrm{S}, 2}(\alpha)>0 \\ P_{m}, & P_{\mathrm{J}_{w(\alpha)}}^{\mathrm{opt}}(\alpha)<P_{m}, R_{\mathrm{S}, 2}(\alpha)>0 \\ P_{\mathrm{J}_{w(\alpha)}}^{\mathrm{opt}}(\alpha), & P_{m} \leq P_{\mathrm{J}_{w(\alpha)}}^{\mathrm{opt}}(\alpha) \leq P_{M}, \\ & R_{\mathrm{S}, 2}(\alpha)>0 \\ P_{\mathrm{J}_{w(\alpha)}}^{\mathrm{opt}}(\alpha), & R_{\mathrm{S}, 2}(\alpha)=0,\end{cases}
$$

where $P_{\mathrm{J}_{w(\alpha)}}^{\mathrm{opt}}(\alpha)$ is given by (8), while, for $R_{\mathrm{S}, 2}(\alpha)>0, P_{m}$ and $P_{M}, P_{m} \leq P_{M}$, are the roots of the quadratic equation $R_{\mathrm{S}}\left(\alpha, P_{\mathrm{J}_{w(\alpha)}}\right)=R_{\mathrm{S}, 2}(\alpha)$.

Proof: It suffices to show that (13) is the solution of the winning jammer's $\mathrm{J}_{w(\alpha)}$ utility maximization problem over $P_{\mathrm{J}_{w(\alpha)}}$ under the constraint $R_{\mathrm{S}}\left(\alpha, P_{\mathrm{J}_{w(\alpha)}}\right) \geq R_{\mathrm{S}, 2}$. This maximization problem is also illustrated in Figure 4. If $R_{\mathrm{S}, 2}(\alpha)=$ 0 , the winner is free to choose any transmission power, as it is not required to improve the secrecy rate. Thus, the winner chooses the power that maximizes its utility $P_{\mathrm{J}_{w(\alpha)}}^{\mathrm{opt}}(\alpha)$, as given in 8 . For $R_{\mathrm{S}, 2}(\alpha)>0$, the values $P_{m}$ and $P_{M}$, $P_{m} \leq P_{M}$, correspond to the winning bidder's transmission powers that yield exactly the second-best bid, $R_{\mathrm{S}, 2}(\alpha)$, as seen in Figure 4. If $P_{m} \leq P_{\mathrm{J}_{w(\alpha)}}^{\mathrm{opt}}(\alpha)$ or $P_{M} \geq P_{\mathrm{J}_{w(\alpha)}}^{\mathrm{opt}}(\alpha)$, the jammer $\mathrm{J}_{w(\alpha)}$ chooses $P_{m}$ or $P_{M}$, respectively, to maximize its utility under the constraint of maintaining the secondbest bid $R_{\mathrm{S}, 2}(\alpha)$. If $P_{m} \leq P_{\mathrm{J}_{w(\alpha)}}^{\mathrm{opt}}(\alpha) \leq P_{M}$, the winning bidder can exploit modification in Definition 1 , and chooses the transmission power $P_{\mathrm{J}_{w(\alpha)}}^{\mathrm{opt}}(\alpha)$, given by 8 , to maximize its utility.

Notice in Theorem 2 and Figure 4 that for the case $P_{m} \leq P_{\mathrm{J}_{w(\alpha)}}^{\mathrm{opt}}(\alpha) \leq P_{M}$, the alteration of Vickrey principle

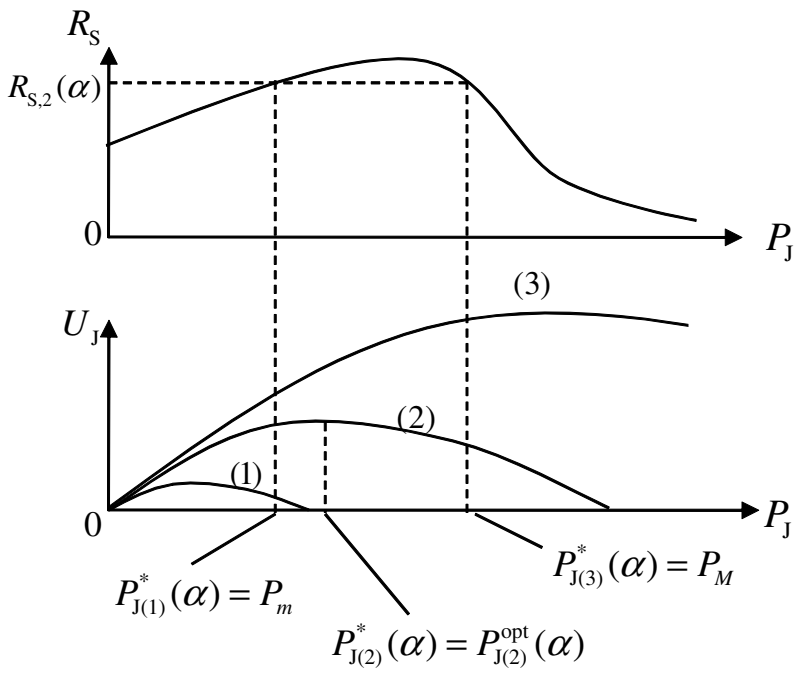

Figure 4. Illustration of Theorem 2.

introduced by Definition 1 brings improvement to both the secrecy rate $R_{\mathrm{S}}$ and the jammer utility $U_{\mathrm{J}}$.

To conclude this section, the outcome of the auction, as given by (13), constitutes the follower's response in the Stackelberg framework and the Stackelberg equilibrium thus reads

$$
\alpha^{*}=\arg \max _{\alpha} R_{\mathrm{S}}\left(\alpha, P_{\mathrm{J}_{w(\alpha)}}^{*}(\alpha)\right) .
$$

Notice that this model essentially requires that the source can anticipate the outcome of the auction game, and thus to be aware of all channel parameters in the system. An alternative to this requirement is for the source to perform a series of auctions with different $\alpha$ and determine the most contributing one. As for the jamming nodes, a jammer $\mathrm{J}_{i}$ needs the knowledge of the parameters $h_{\mathrm{SD}}$ and $h_{\mathrm{SE}}$, in addition to $h_{\mathrm{J}_{i i}}$. On the positive side, a bidder requires no information on number of other bidders, their strategies or channel parameters, which is in line with the basic Vickrey implications.

\section{Power Control Scheme}

In this section, a scheme is proposed that allows the source to employ multiple cooperative jammers simultaneously. Besides setting the fraction $\alpha$, the source also communicates to the jammers the set $\mathcal{J} \subseteq\{1, . ., N\}$ of chosen jammers. During their data transmission phase of duration $1-\alpha$, the participating cooperative jammers, i.e., the jammers from the set $\mathcal{J}$, share the communications resource and their transmission scheme results in an interference channel [17] [13] [18]. Though in general information theoretically suboptimal, we shall assume that interference is treated as noise for our setting [19] [20], which is the standard assumption for a gametheoretic approach to an interference channel [13] [18]. In terms of a Stackelberg framework, the follower's response is the set of powers that is the outcome of a power control game the jammers play, in the form of a Nash equilibrium, as detailed in Section V-B. 


\section{A. Communications Model}

Under the setting described above, the source's secrecy rate (1) now becomes:

$$
\begin{aligned}
R_{\mathrm{S}}\left(\alpha, \mathcal{J}, P_{\mathrm{J}_{i \in \mathcal{J}}}\right)= & \alpha\left[\log _{2}\left(1+\frac{h_{\mathrm{SD}} P_{\mathrm{S}} / \alpha}{\sigma^{2}+\sum_{i \in \mathcal{J}} h_{\mathrm{J}_{i} \mathrm{D}} P_{\mathrm{J}_{i}}}\right)-\right. \\
& \left.\log _{2}\left(1+\frac{h_{\mathrm{SE}} P_{\mathrm{S}} / \alpha}{\sigma^{2}+\sum_{i \in \mathcal{J}} h_{\mathrm{J}_{i} \mathrm{E}} P_{\mathrm{J}_{i}}}\right)\right]
\end{aligned}
$$

The utility of the chosen jammers $\mathrm{J}_{i \in \mathcal{J}}$, similar to (2), is given by

$$
\begin{gathered}
U_{\mathrm{J}_{i \in \mathcal{J}}}\left(\alpha, \mathcal{J} ; P_{\mathrm{J}_{i}}\right)= \\
(1-\alpha) \log \left(1+\frac{h_{\mathrm{J}_{i i}} P_{\mathrm{J}_{i}}}{\sigma^{2}+\sum_{l \in \mathcal{J}, l \neq i} h_{\mathrm{J}_{l i}} P_{\mathrm{J}_{l}}}\right)-c P_{\mathrm{J}_{i}},
\end{gathered}
$$

with $P_{\mathrm{J}_{i}} \leq \bar{P}_{\mathrm{J}_{i}}$.

\section{B. Game-Theoretic (Power-Control) Model}

According to the Stackelberg framework in Section III-B, the rational and selfish jammers $\mathrm{J}_{i}$ are aware of the parameters $\alpha$ and $\mathcal{J}$ set by the source. Competition between chosen jammers is formulated as a non-cooperative power control game [13] [18] in the interference channel during the jammers' data transmission phase of duration $1-\alpha$, with their utilities and power/strategy spaces provided in Section V-A. Each jammer $\mathrm{J}_{i}$ chooses its strategy $P_{\mathrm{J}_{i}}$ in order to maximize its utility (16), aware that this decision will affect the other jammers' strategies. The outcome of the game $P_{\mathrm{J}_{i \in \mathcal{J}}}^{*}(\alpha, \mathcal{J})$ can be described by the game-theoretic concept Nash equilibrium (NE), defined as the state whereby any unilateral deviation in player's strategy would not produce any gain [15]. Herein, NE can be conveniently obtained as a fixed point of the best responses [15] of the participating jammers. In particular, the best response of each jammer can be found by setting the derivative of (16) with respect to $P_{\mathrm{J}_{i}}$ to zero, i.e., $\partial U_{\mathrm{J}_{i}} /\left.\partial P_{\mathrm{J}_{i}}\right|_{P_{\mathrm{J}_{j \in \mathcal{J}}, j \neq i}=P_{\mathcal{J}_{j} \in \mathcal{J}, j \neq i}^{*}}=0$, and the NE is given by the following set of $|\mathcal{J}|$ equations:

$$
\begin{gathered}
P_{\mathrm{J}_{i \in \mathcal{J}}}^{*}(\alpha, \mathcal{J})= \\
{\left[\frac{1-\alpha}{c \ln 2}-\frac{\sigma^{2}}{h_{\mathrm{J}_{i i}}}-\sum_{l \in \mathcal{J}, l \neq i} \frac{h_{\mathrm{J}_{l i}}}{h_{\mathrm{J}_{i i}}} P_{\mathrm{J}_{l}}^{*}(\alpha, \mathcal{J})\right]_{0}^{\bar{P}_{\mathrm{J}_{i}}} .}
\end{gathered}
$$

Clearly, the game has a unique NE if the system (17) has a unique solution. This power control game has been discussed in [13] and for the more general framework of wideband systems in [18], where it was shown that the NE always exists and that it is unique if the interference matrix $\mathbf{H}$, defined as $[\mathbf{H}]_{l i}=h_{J_{l i}}$, is strictly diagonally dominant, i.e., $\sum_{l \in \mathcal{J}, l \neq i} h_{\mathrm{J}_{l i}} / h_{\mathrm{J}_{i i}}<1$. This condition for uniqueness of the $\mathrm{NE}$ is intuitive since it simply imposes an upper bound on the interference: in fact, with negligible interference, equations (17) become decoupled and the solution clearly exists and is unique. In the remainder of this work, we assume that this condition is satisfied.
To conclude, the Stackelberg equilibrium is given as

$$
\begin{gathered}
\left(\alpha^{*}, \mathcal{J}^{*}\right)=\arg \max _{(\alpha, \mathcal{J})} R_{\mathrm{S}}\left(\alpha, \mathcal{J}, P_{\mathrm{J}_{i \in \mathcal{J}}^{*}}^{*}(\alpha, \mathcal{J})\right) \\
\text { s.t. } 0<\alpha \leq 1, \mathcal{J} \subseteq\{1, \ldots, N\}
\end{gathered}
$$

where $P_{\mathrm{J}_{i \in \mathcal{J}}}^{*}(\alpha, \mathcal{J})$ are the $|\mathcal{J}|$ power responses given by (17). Notice that the source needs to be aware of all the instantaneous channel power gains in the system, while the cooperative jammers are required to know $h_{\mathrm{J}_{i l}}, i, l \in \mathcal{J}$. Finally, it should be clear that for $N=1$ the power control scheme boils down to the reference Stackelberg scheme described in Section III-B.

\section{NUMERICAL RESUlts}

In this section, we provide further insights into the proposed mechanisms using numerical results. The S-D pair secrecy rate $R_{\mathrm{S}}$ and the jammer's utility $U_{\mathrm{J}}$ are illustrated in Figure 5-(a) and Figure 5-(b), respectively, for the reference Stackelberg model described in Section III, as a function of the jammer's location $(x, y)$. Positions of the nodes $\mathrm{S}, \mathrm{D}$ and $\mathrm{E}$ are indicated in the figure, while the jammer's intended destination $D_{J}$ is placed $d=3[\mathrm{~m}]$ from the jammer J. A simple path-loss model with the propagation factor $\gamma=2$ is used, with $P_{\mathrm{S}}=\bar{P}_{\mathrm{J}}=10$ [Watt], $\sigma^{2}=1$ [Watt] and $c=0.25$ [bit $/ \mathrm{sec} / \mathrm{Hz} /$ Watt]. Since the nodes $\mathrm{D}$ and $\mathrm{E}$ are equally distanced from the source $\mathrm{S}$, the secrecy rate $R_{\mathrm{S}}$ without jamming is zero. Secrecy rate is largest when the jammer is in the eavesdropper's E vicinity, and zero when the jammer is closer to the destination than to the eavesdropper. The latter also holds for the jammer's utility $U_{\mathrm{J}}$. Interestingly, when the jammer is close to the eavesdropper, its utility is very small, as the source needs a relatively small jamming power and preserves the majority of the bandwidth $\alpha$ for itself.

The source's secrecy rate and the utility of a chosen cooperative jammer in Stackelberg equilibria, averaged over channel realizations under assumption of independent blockfading, $\mathbb{E}\left[R_{\mathrm{S}}\right]$ and $\mathbb{E}\left[U_{\mathrm{J}}\right]$, respectively, are shown in Figure 6 as functions of the number of potential jamming nodes $N$, for the three proposed schemes and parameters $P_{\mathrm{S}}, \bar{P}_{\mathrm{J}}, \sigma^{2}$ and $c$ chosen as above, with $\mathbb{E}\left[h_{\mathrm{SD}}\right]=\mathbb{E}\left[h_{\mathrm{SE}}\right]=\mathbb{E}\left[h_{\mathrm{J}_{i} \mathrm{E}}\right]=$ $\mathbb{E}\left[h_{\mathrm{J}_{i} \mathrm{D}}\right]=\mathbb{E}\left[h_{\mathrm{J}_{i i}}\right]=0 \mathrm{~dB}$ and $\mathbb{E}\left[h_{\mathrm{J}_{i l}}\right]=-10 \mathrm{~dB}, i, l=$ $1, . ., N, i \neq l$. Small values for jammers' interference channel gains favor the uniqueness of Nash equilibrium for the power control game, as discussed in Section V. Both the secrecy rate and a chosen jammer's utility benefit with the proposed mechanisms. The secrecy rate increases with $N$, with the power control scheme, described in Section V, outperforming the auction scheme, in Section IV, due to multiple simultaneous jamming transmissions, while the auction outperforms the multi-jammer Stackelberg scheme, in Section III, due to competitiveness. The opposite relations between the three schemes hold for $U_{\mathrm{J}}$, although the cumulative utility of the chosen jammers for the power control scheme $\sum_{i \in \mathcal{J}} U_{\mathrm{J}_{i}}$ is the largest as multiple jamming nodes are allowed to transmit data. Notice that $U_{\mathrm{J}}$ for the power control scheme decreases for $N \geq 4$ jammers due to an increased interference. It is also noted that for a small $N$, the probability of having a contributing jammer is small, 


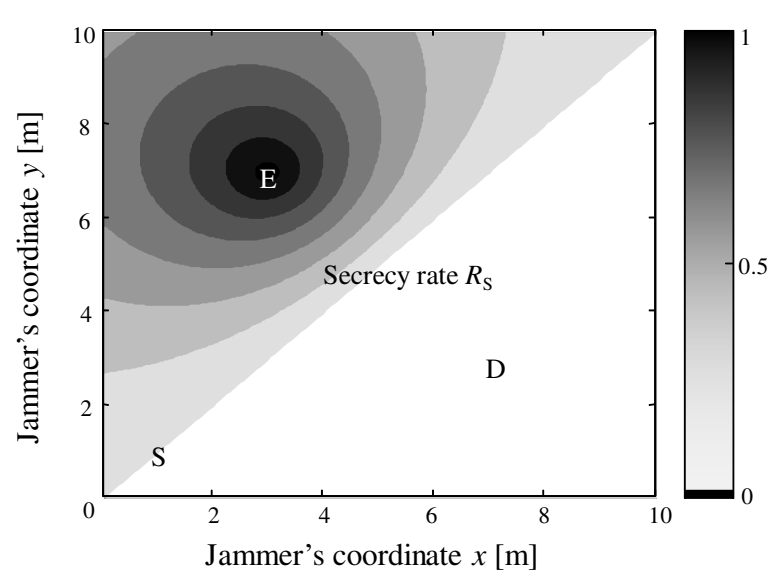

(a)

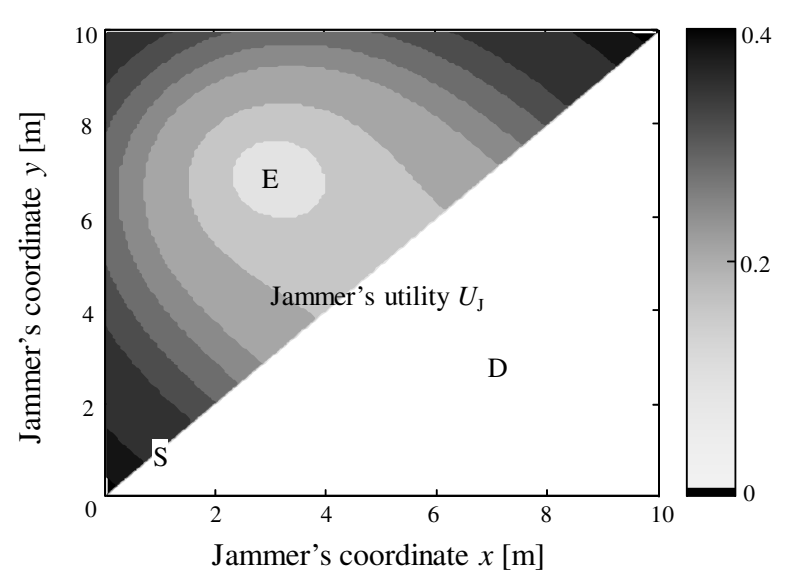

(b)

Figure 5. Secrecy rate $R_{\mathrm{S}}$ and cooperative jammer's utility $U_{\mathrm{J}}$ as functions of jammer's location, for the reference Stackelberg scheme $(N=1)$.

frequently yielding zero jammer's utility and thus relatively small average utilities for any of the schemes.

\section{CONCLUDING REMARKS}

In this paper, we have proposed a game-theoretic framework for enhancing secret communications in wireless channel with a cooperative jamming assistance of non-altruistic users. The framework is based on the spectrum leasing concept, wherein a source-destination pair communicating messages that need to be kept secret from an eavesdropping node, is willing to compensate external potential cooperative jammer(s) with a fraction of its bandwidth. Interaction between the nodes is based on the Stackelberg concept and, for a multi-jammer scenario, also deploys auctioning and power control game. $\mathrm{Nu}$ merical results corroborate the benefits for all involved nodes, despite their selfish nature. Future work includes investigating the impact of multiple antennas deployed at the jammer nodes.

\section{REFERENCES}

[1] C. Shannon, "Communication Theory of Secrecy Systems," Bell Syst. Tech. Journal, vol. 28, no. 4, pp. 656-715, Sep. 1949.

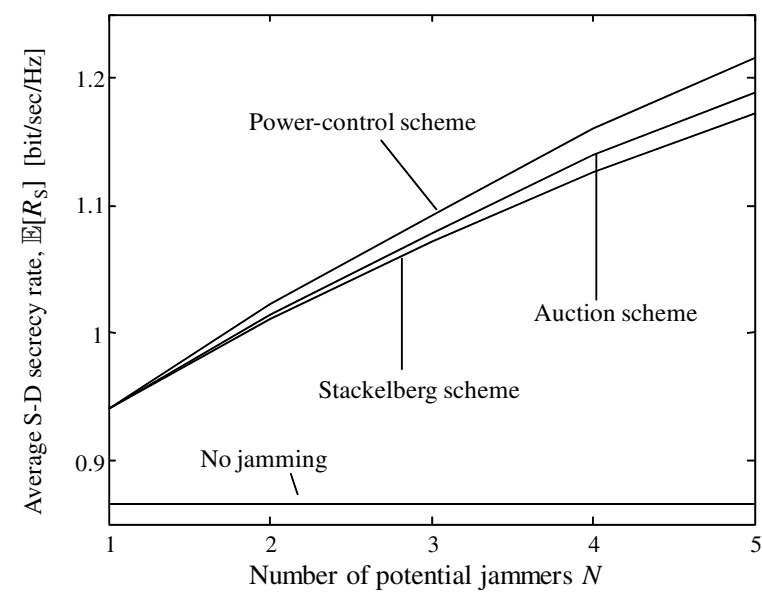

(a)

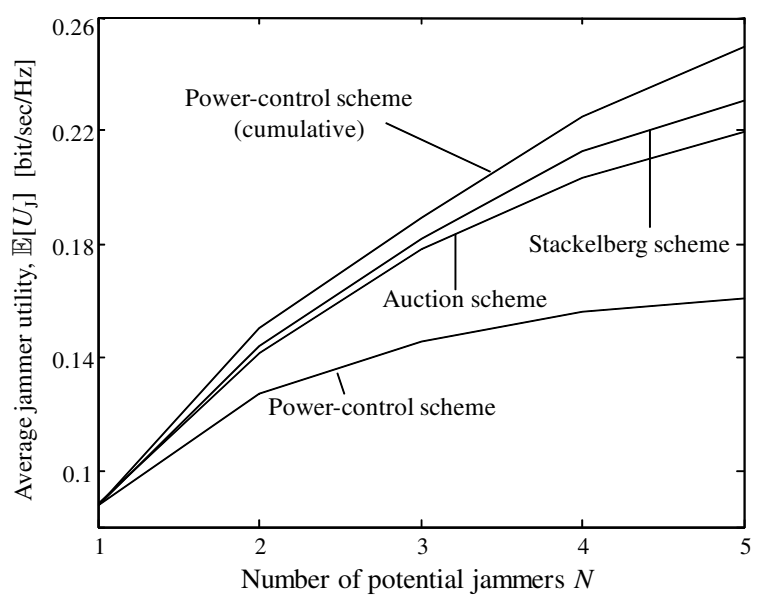

(b)

Figure 6. Average secrecy rate $\mathbb{E}\left[R_{\mathrm{S}}\right]$ and winning cooperative jammer's utility $\mathbb{E}\left[U_{\mathrm{J}}\right]$ versus the number of potential cooperative jammers.

[2] A. D. Wyner, "The Wire-Tap Channel," Bell Syst. Tech. Journal, vol. 54, no. 8, pp. 1355-1387, Oct. 1975.

[3] I. Csiszar and J. Komer, "Broadcast Channels with Confidential Messages," IEEE Trans. Inf. Theory, vol. 24, no. 3, pp. 339-348, May 1978.

[4] S. Leung-Yan-Cheong and M. Hellman, "The Gaussian Wire-tap Channel," IEEE Trans. Inf. Theory, vol. 24, no. 4, pp. 451-456, July 1978.

[5] E. Tekin and A. Yener, "The Multiple Access Wire-Tap Channel: Wireless Secrecy and Cooperative Jamming," in Proc. Inf. Theory Applications Workshop, San Diego, CA, Jan. 2007.

[6] E. Tekin and A. Yener, "The General Gaussian Multiple Access and Two-Way Wire-Tap Channels: Achievable Rates and Cooperative Jamming," IEEE Trans. Inf. Theory, vol. 54, no. 6, pp. 2735 - 2751, June 2008.

[7] L. Lai and H. El Gamal, "The Relay-Eavesdropper Channel: Cooperation for Secrecy," IEEE Trans. Inf. Theory, vol. 54, no. 9, pp. 4005-4019, Sep. 2008.

[8] X. Tang, R. Liu, P. Spasojevic, and H. V. Poor, "The Gaussian Wiretap Channel With a Helping Interferer," in Proc. IEEE Int. Symp. Inf. Theory, Toronto, Canada, July 2008.

[9] X. He and A. Yener, "Providing Secrecy With Structured Codes: Tools and Applications to Two-User Gaussian Channels," IEEE Trans. Inf. Theory, July 2009, submitted for publication.

[10] E. Tekin and A. Yener, "Achievable Rates for the General Gaussian Multiple Access Wire-Tap Channel with Collective Secrecy," in Proc. Allerton Conf. Commun. Control Comput., Monticello, IL, Sep. 2006.

[11] X. He and A, Yener, "Two-hop Secure Communication Using an Untrusted Relay,” EURASIP Jour. Wireless Commun. Netw., Special 
Issue on Wireless Physical Layer Security, vol. 2009, Article ID 305146, 13 pages, 2009. doi:10.1155/2009/305146.

[12] E. Ekrem and S. Ulukus, "Secrecy in Cooperative Relay Broadcast Channels," IEEE Trans. Inf. Theory, Oct. 2008, submitted for publication.

[13] O. Simeone, I. Stanojev, S. Savazzi, Y. Bar-Ness, U. Spagnolini and R. Pickholtz, "Spectrum Leasing to Cooperating Secondary Ad Hoc Networks," IEEE Jour. Select. Areas Commun., vol. 26, no. 1, pp. 203213, Jan. 2008.

[14] I. Stanojev, O. Simeone, U. Spagnolini, Y. Bar-Ness and R. Pickholtz, "Cooperative ARQ via Auction-Based Spectrum Leasing," IEEE Trans. Commun., vol. 58, no. 6, pp. 1843 - 1856, June 2010.

[15] M. J. Osborne and A. Rubenstein, A Course in Game Theory, MIT Press, 1994.

[16] P. Klemperer, "Auction Theory: A Guide to the Literature," J. Economics Surveys, vol. 13, no. 3, pp. 227-286, Jul. 1999.

[17] T. S. Han and K. Kobayashi, "A New Achievable Rate Region for the Interference Channel," IEEE Trans. Inf. Theory, vol. 27, no. 1, pp. 49-60, Jan. 1981.

[18] G. Scutari, D. P. Palomar and S. Barbarossa, "Optimal Linear Precoding/ Multiplexing for Wideband Optimal Linear Precoding Strategies for Wideband Noncooperative Systems Based on Game Theory-Part I: Nash Equilibria," IEEE Trans. on Signal Process., vol. 56, no. 3, pp. 1230 - 1249, Mar. 2008.

[19] X. Shang, G. Kramer and B. Chen, "A New Outer Bound and the NoisyInterference Sum-Rate Capacity for Gaussian Interference Channels," IEEE Trans. Inf. Theory, vol. 55, no. 2, pp. 689 - 699, Feb. 2009.

[20] V. S. Annapureddy and V.V. Veeravalli, "Gaussian Interference Networks: Sum Capacity in the Low Interference Regime and New Outer Bounds on the Capacity Region," IEEE Trans. Inf. Theory, vol. 55, no.7, pp. 3032-3050, July 2009.

[21] J. O. Neel, Analysis and Design of Cognitive Radio Networks and Distributed Radio Resource Management Algorithms, PhD. dissertation, Virginia Polytechnic Institute, September 2006.

[22] R. M. Corless, G. H. Gonnet, D. E. Hare, D. Jeffrey and D. E. Knuth, "On the Lambert W function," Adv. in Computational Math., vol. 5, 1996.

[23] W. Vickrey, "Counterspeculations, Auctions, and Competitive Sealed Tenders," Journal of Finance, vol. 16, pp. 8-37, 1961.

[24] B. Niu, O. Simeone, O. Somekh and A. M. Haimovich, "Ergodic and Outage Performance of Fading Broadcast Channels with 1-Bit Feedback," IEEE Trans. Veh. Technol., vol. 59, no. 3, pp. 1282 - 1293, Mar. 2010.

[25] D. Zhang, R. Shinkuma and N. B. Mandayam, "Bandwidth Exchange: An Energy Conserving Incentive Mechanism for Cooperation," IEEE Trans. Wireless Commun., vol. 9, No. 6, pp. 2055-2065, June 2010.

[26] M. Lindstrom and P. Lungaro, "Resource Delegation and Rewards to Stimulate Forwarding in Multihop Cellular Networks," in Proc. IEEE Veh. Technol. Conference VTC, Stockholm, Sweden, June 2005.

[27] Z. Zhang, J. Shi, H.-H. Chen, M. Guizani, and P. Qiu, "A Cooperation Strategy Based on Nash Bargaining Solution in Cooperative Relay Networks," IEEE Trans. Veh. Technol., vol. 57, no. 4, pp. 2570-2577, July 2008 . 